\title{
Rapports des jeunes au travail, pratiques d'emploi et diplômes
}

\section{L'amalgame de parcours différenciés}

María Eugenia Longo

\section{INTRODUCTION}

À partir des années 1980, plusieurs études ont mis en évidence la différenciation croissante des parcours professionnels des jeunes, et cela dans des contextes sociétaux différents (Roberts et al., 1994 ; EGRIS, 2000). Jérôme Gautié (2003) a montré que cette différenciation est observable à travers la multiplication des transitions, c'est-à-dire des passages d'un état à un autre (emploi/chômage, inactivité/emploi, emploi/formation, formation/chômage), à travers l'individualisation des trajectoires, c'est-à-dire la diversification des états et transitions à partir d'une même origine sociale ou visant à atteindre une même situation professionnelle, et à travers la difficulté croissante à aborder les transitions en raison de la diversification des situations et du flou de leurs frontières. Le phénomène de différenciation est lié aux emplois plus précaires et instables, notamment chez les nouvelles générations (Jacinto, Chitarroni, 2010), ainsi qu'aux politiques publiques qui créent parfois des positions ambiguës sur le marché du travail pour pallier ces problèmes (Lima, 2016 ; Longo, 2016a). La différenciation a également été appréhendée dans un cadre d'accroissement des inégalités liées à l'origine sociale ou au diplôme et d'émergence de nouvelles sortes d'inégalités (Dubet et al., 2010 ; Dubet, 2000).

Cependant, les différences entre les parcours ${ }^{1}$ sont loin de se réduire à de simples effets de contexte ou uniquement aux origines sociales ou éducatives des individus. La différenciation des parcours comporte la subjectivité, une composante de singularisation liée aux arbitrages et aux choix des acteurs. Quoique issues de la socialisation, les pratiques sont le produit des dispositions ouvertes et créatrices de sens (Lahire, 1998). Cette composante

1. Comme nous l'avons développé dans un article à part entière (Longo, 2016b), nous préférons le terme « parcours » à d'autres - comme « itinéraire », «trajectoire », «navigation »- pour y référer le produit croisé des décisions subjectives et des limites objectives du cheminement contraint par les institutions (Passeron, 1990). 
Rapports des jeunes au travail, pratiques d'emploi et diplômes

peut être examinée à la lumière des rapports au travail et à l'emploi, qui interagissent avec d'autres facteurs pour renforcer parfois leurs effets ou au contraire pour les atténuer.

L'intérêt pour ces rapports n'est pas nouveau et se retrouve au cœur d'enquêtes nationales et internationales de premier plan (Baudelot, Gollac, 2003 ; SPREW, 2008 ; Mercure, Vultur, 2010). Cependant, ces différents travaux ne parviennent pas toujours à mettre en exergue la multiplicité ou l'articulation des différentes dimensions liées à la vie professionnelle lactivité, travail, emploi, marché du travail), ni à relier les perceptions et les pratiques effectives des individus lors du déroulement du parcours.

Cet article vise à souligner la pertinence d'analyser la subjectivité associée au travail, dans le contexte actuel de différenciation des parcours, à travers la notion de rapports à la vie professionnelle. Cette notion issue d'une enquête sur les parcours des jeunes en Argentine a également été mise à l'épreuve dans d'autres contextes, comme ceux français et québécois (Longo, 2014 ; Longo, Bourdon, 2016). Elle permet à la fois de multiplier les dimensions considérées (le rapport à l'activité, le rapport au travail, le rapport à l'emploi et le rapport au monde professionnell et de les réunir sous un même objet. Dans un premier temps, nous décrirons ces rapports en retraçant quelques antécédents de cette notion et en illustrant leurs modalités avec les données de notre enquête. Ces rapports doivent également être analysés dans une perspective dynamique. Enfin, nous arriverons à l'objectif principal de l'article, soit une description des interactions des sens et des pratiques, voire des rapports à la vie professionnelle et à l'insertion effective dans l'emploi, qui nous permettra d'interroger l'origine sociale et le diplôme des jeunes enquêtés.

Les données qui fondent cet article sont issues d'une recherche qualitative et longitudinale menée entre 2006 et 2011 (trois vagues d'enquête tous les deux $a^{2}{ }^{2}$ ) sur les parcours de 84 jeunes hommes et femmes 3 âgés de 16 à 19 ans ayant suivi différents types de formation en Argentine. Ces données résultent de deux outils de terrain : des entretiens semi-directifs et des calendriers biographiques. Les entretiens comportent des récits sur les parcours professionnels et scolaires, sur les représentations sur le travail, les projets de

2. L'enquête a démarré par un sondage court - questionnaire standardisé - auprès de 422 jeunes finissant leurs études dans les institutions scolaires séléctionnées. Certains de ces jeunes - ceux qui ont manifesté leur volonté à s'engager dans une enquête à long terme - ont été appelés pour une première vague d'entretiens approfondis réalisés en 2006. Au départ, on comptatit 84 jeunes, 79 d'entre eux ont été interrogés ensuite en 2008 et 50 en 2011. Les exemples utilisés ici se concentreront sur les deux premières vagues d'enquête où le nombre des effectifs est resté plutôt stable.

3. Contre toute simplification apparente due à l'établissement d'un segment d'âge, depuis la mise en œuvre de l'enquête, nous défendons une définition identitaire, statutaire socialement construite et aussi chronologique de la jeunesse. Nous avons développé cette définition et son opérationnalisation dans Longo, 2016 c. 
vie, de même que sur la résidence, la famille, les temporalités, les relations, les temps forts biographiques et le devenir adulte. Les calendriers biographiques rassemblent des informations factuelles synthétiques et objectivées, mois par mois, sur différents états et événements des parcours lors du suivi. «C'est dans les descriptions précises du travail et dans ce que les exécutants en disent qu'il faut chercher ces significations » (Bernoux, 2016, p. 32). La complémentarité des méthodes pour associer l'aspect symbolique et l'aspect pratique des rapports a été donc recherchée.

De plus, l'enquête ayant pour but l'étude des rapports au travail et au temps des jeunes avait pour hypothèse de départ l'incidence du poids de la formation sur les cheminements. Les jeunes qui ont participé à l'enquête avaient ainsi en commun le fait de terminer leur formation - c'est donc dire qu'ils se situaient à un tournant de leur parcours de vie - et ils se différenciaient par le type de formation suivie en Argentine : le secondaire appelé « polimodal » en Argentine (correspondant au baccalauréat économique et social en France), le secondaire « technique » (semblable aux filières de baccalauréat professionnel en Francel et la «formation professionnelle », c'est-à-dire des formations courtes pour des jeunes qui ne terminent pas leurs études primaires ou secondaires. Cette dernière catégorie définit les jeunes sans diplôme de l'enquête. De plus, les différences entre les filières s'accentuent selon le secteur de gestion (public ou privé) de l'institution qui les propose. En effet, le secteur est fréquemment associé à différentes origines sociales : les jeunes issus de la formation professionnelle se caractérisent par une origine sociale populaire, ceux issus du secondaire technique public et du secondaire polimodal public par une origine sociale populaire ou moyenne, et ceux issus du secondaire technique et polimodal privé par une origine sociale moyenne, certains provenant même de classes sociales aisées. Ces différences illustrent la problématique de la fragmentation sociale et éducative des parcours des jeunes en Argentine (Miranda, 2006) [voir encadré, p. 70].

\section{LES « RAPPORTS À LA VIE PROFESSIONNELLE » : ANTÉCÉDENTS ET DIMENSIONS D'UN SEUL OBJET}

Si pour les travailleurs moins qualifiés ou situés au bas de l'échelle sociale, la précarisation et l'informalité de l'emploi découlent des possibilités qui s'offrent à eux, aux transitions professionnelles involontaires comme cellesci s'en ajoutent d'autres, volontaires, associées à un « désir de transition » (Gautié, 2003). Il existe différents usages sociaux d’une même catégorie d'emploi, en fonction des représentations liées au travail. On observe également une recomposition des étapes de la vie active et du cycle professionnel selon le sens donné au travail (Bidart, Longo, 2007). Certains moments, qui étaient auparavant considérés comme des actes ponctuels ll'entrée et la sortie de la vie active), deviennent depuis quelques décennies, pour les 
Dossier

Rapports des jeunes au travail, pratiques d'emploi et diplômes

Argentine : un cas de va-et-vient dans la régulation du marché du travail

L'Argentine se différencie de ses pairs latino-américains par la construction progressive, après la Seconde Guerre mondiale, d'une structure salariale homogène et stable. Cependant, les dictatures politiques des années 1970 et les gouvernements néolibéraux des années 1990 ont introduit des réformes directes de la structure de l'État et la dérégulation des protections sociales, avec de graves conséquences : chômage, instabilité et précarité de l'emploi, qui sont plus accentués dans la population juvénile notamment. Ces politiques ont débouché sur une crise économique, sociale et politique survenue en 2001 , lors de laquelle le chômage a atteint 20 \% dans le pays (Perez, 2008). Malgré la diminution de ce chiffre par la suite, la probabilité des jeunes d'être au chômage est restée trois fois plus forte que pour les adultes (MTEySS, 2005). De plus, ce sont les jeunes, les femmes et les petits salariés qui sont les populations les plus affectées par un fort turn over entre emplois. L'insertion professionnelle juvénile est aussi profondément touchée par d'autres difficultés : $85 \%$ des jeunes Argentins commencent leur vie active dans le secteur des emplois non déclarés - le travail informel - sans aucune protection ou sécurité d'emploi (Salvia, Tuñon, 2003). Ce début d'insertion se maintient ensuite largement pour la plupart des jeunes actifs. Le MTEySS* signalait en 2007 que $62 \%$ des personnes actives âgées de 18 à 24 ans travaillaient dans des emplois non déclarés. Ces données sont préoccupantes car on constate également la précocité de l'insertion là l'âge de 15 ans pour les jeunes hommes et de 17 ans pour les jeunes femmes) et la simultanéité d'exercice d'un travail et des études, souvent en dépit de la permanence future dans les études. Enfin, ces tendances se renforcent encore davantage au fur et à mesure que le niveau éducatif et l'origine sociale des jeunes diminuent. D'une part, si le diplôme secondaire est nécessaire, il n'est pas suffisant pour assurer l'accès à l'emploi ni pour permettre d'obtenir un emploi à caractère formel (Filmus et al., 2001). D'autre part, l'origine sociale semblerait avoir un poids plus important que le diplôme ; elle possède en effet des effets directs et indirects sur les possibilités d'insertion professionnelle : elle détermine les parcours de formation et, à diplôme égal, les jeunes de différentes origines sociales n'accèdent pas aux mêmes offres d'emploi (Perez et al., 2016).

L'enquête démarre en 2003 au moment où de nouvelles régulations visaient à compenser la flexibilisation du travail des années 1990, en particulier pour les jeunes possédant des qualifications moindres et de faibles possibilités d'employabilité. Pour cette population, une nouvelle réforme éducative et des politiques sociales et d'emploi mettent l'accent sur l'éducation formelle, les systèmes de formation professionnelle, les programmes publics actifs de capacitation et d'emploi, l'employabilité et la socialisation des jeunes au travail. Ces politiques créent sans conteste de l'inclusion sociale, mais le caractère informel du travail perdure chez les jeunes Argentins. En effet, la proportion de jeunes salariés non couverts par la sécurité sociale est passée de $72 \%$ en 2003 à $58 \%$ en 2008. Si on ajoute à cet indicateur le nombre de jeunes travailleurs non salariés sans sécurité sociale, le taux total est descendu de $79 \%$ à $65 \%$ entre 2003 et 2008, mais il est remonté à $69 \%$ en 2010 (Salvia, 2013).

* Ministerio de Trabajo, Empleo y Seguridad Social de Argentina (ministère du travail, de l'emploi et de la sécurité sociale d'Argentine). 
institutions et les individus, des «phases de transition » de durées variables, avec une forte responsabilisation de l'individu dans la construction du sens de sa trajectoire (Dubar, 1994).

En considérant le rôle clé de la subjectivité, toute une série de recherches et de réflexions sociologiques très riches sur les significations attribuées au travail se développe depuis plusieurs décennies (Paugam, 2000 ; NicoleDrancourt, Roulleau-Berger, 2001 ; Baudelot, Gollac, 2003 ; Antunes, 2005 ; Delay, 2008 ; Mercure, Vultur, 2010). Ces recherches abordent la subjectivité au travail à travers des « prismes » différents (Loriol, 2017) sans avoir toujours l'intérêt ou l'objectif de réintégrer les dimensions variées qui émergent dans les données empiriques. De plus, la difficulté dans les enquêtes d'approcher cette question de manière directe (Méda, Vendramin, 2013) mène à construire des oppositions apparentes dans le rapport au travail (centralité/ marginalité ; importance absolue/relative ; rétribution/épanouissement ; instrumentalité/expressivitél au lieu de mettre en évidence l'assemblage ambigu de dimensions distinctes de la vie professionnelle. Nous synthétiserons dans les lignes qui suivent celles qui sous-tendent la notion de rapports à la vie professionnelle ressortant empiriquement du terrain argentin et visant à dépasser ces défis4.

\section{Le rapport à l'activité professionnelle : continuum d'importance et de priorité}

Parmi les notions les plus anciennes, celle du rapport à l'activité de Chantal Nicole-Drancourt (1992) comme « la position de l'individu envers l'option professionnelle » permet de bien comprendre ce qui détermine les stratégies d'engagement professionnel dans un sens large. Suivant la conception de cette auteure, ce rapport s'inscrit dans l'imaginaire et il semble être indépendant des opportunités d'emploi réelles. Il s'agit d'une dimension antérieure à celles qui suivent ici dans la mesure où elle positionne le jeune au-delà de la façon d'exercer concrètement ses emplois. Elle définit la base d'un ethos du travail (Mercure, Vultur, 2010) guidant les autres choix liés à l'aspect professionnel. Toutefois, elle s'éloigne ici de l'activité en tant que travail effectif « en train de se faire » (Eckert, Vultur, 2016) - davantage relié aux rapports au travail et à l'emploi que nous verrons par la suite -, pour tendre vers une notion élargie de l'action d'une personne dans la sphère professionnelle.

Dans notre recherche, le rapport à l'activité professionnelle est lié à la place du travail dans la vie des jeunes. Cependant, la caractérisation de ce rapport

4. Les résultats qui seront présentés découlent des pratiques et des perceptions des jeunes. Les dimensions empiriques de la vie professionnelle rencontrent plus d'obstacles si on les limite à un petit nombre de questions et de réponses standardisées fermées. L'ouverture des réponses et l'introduction de nuances servent à mieux saisir ce qui est de l'ordre de l'idéal ou du réel en ce qui concerne l'expérience de travail (Méda, 2010), et ces éléments sont plus féconds dans des approches compréhensives et qualitatives. 
Dossier

Rapports des jeunes au travail, pratiques d'emploi et diplômes

uniquement en fonction de «l'importance » accordée au travail - opérationnalisation la plus fréquente - reste peu probante (Méda, 2010). Les positions des jeunes Argentins forment davantage un continuum selon l'importance absolue (l'activité est une fin en soil et la priorité relative de l'activité professionnelle et des autres sphères de la vie (formation, loisirs, famille, engagements militants) : centralité (ou priorité et importance) - « Le travail est fondamental surtout parce qu'il ordonne ta $v^{2} e^{5}$. » - ; naturalisation lou priorité sans importance) - «En effet on naît, il faut étudier, et puis travailler, travailler jusqu'à la retraite, c'est la loi de la vie ${ }^{6}$. »- ; propension lou importance sans prioritél - « Ma mère m'a dit de me tranquilliser un peu, mais je n'aime pas ne pas travailler. »- ; non-centralité lou sans importance, sans prioritél - « Je n'avais pas envie de travailler, pour moi, avec l'école, ça suffisait". » - ; aversion à l'activité lou sans priorité, rejet) - « Je ne veux pas travailler. Je n'aime pas ça ${ }^{8}$. »

En mettant en relation leur activité professionnelle avec les autres sphères de leur vie (éducative, familiale, récréative, etc.), il est possible d'évaluer les priorités des jeunes. Cette distinction entre sphères est pourtant analytique puisque ces dernières sont imbriquées dans les parcours. De plus, malgré son niveau d'abstraction, ce rapport n'est pas déconnecté de l'action, car la répartition du temps de vie peut se faire en fonction de celui-ci. Enfin, cette première notion manque de précision lorsqu'il s'agit de différencier l'attitude des personnes envers l'emploi en général ou envers une profession spécifique (Dubar, 1994). Il est dès lors intéressant d'effectuer une distinction entre activité, emploi et travail quand on aborde les rapports des jeunes.

\section{Le rapport à l'emploi : choix concrets des conditions d'exercice de l'activité}

Anne-Marie Daune-Richard définit le rapport à l'emploi «comme un rapport à ce qui spécifie l'emploi occupé : contenu de l'emploi, conditions de l'emploi (localisation, type d'entreprise et de rapport en particulier hiérarchique, conditions salariales, contrat de travail), perspectives, expression d'un positionnement dans la hiérarchie sociale » (Daune-Richard, 1992, p. 85). Dans notre recherche, le rapport à l'emploi a été opérationnalisé par les critères mobilisés pour évaluer concrètement les emplois et répondre à la question : qu'est-ce qu'un bon emploi ? Les représentations les plus abstraites du travail sont mises à l'épreuve dans les emplois concrets, l'activité effective. Ces critères se trouvent amalgamés dans les parcours, avec la même importance, ou fortement hiérarchisés, selon les jeunes interviewés.

5. Simon, polimodal privé, vague 2 .

6. Marcelo, technique public, vague 2 .

7. Micaela, technique public, vague 1 .

8. Sofia, polimodal privé, vague 1. 
La sécurité ou la possibilité d'obtenir un emploi déclaré constitue un critère central - « Un vrai emploi ? Quand tu as tout, ils t'embauchent de manière formelle avec mutuelle, avec tout le kit ${ }^{9}$. »; la stabilité ou la permanence (CDI) dans l'emploi - « Je préfère avoir un emploi stable ${ }^{10}$. » - ; la carrière ou les perspectives d'évolution dans l'emploi - «J'ai d'abord accepté, car ils m'ont promis de monter ${ }^{11}$. » - ; la tâche, le contenu du poste, les activités concrètes dans l'emploi - « Je cherche un emploi relié à ce que je suis en train d'étudier ${ }^{12}$. » - ; le salaire - « Un bon emploi veut dire à mon avis une bonne rémunération ${ }^{13}$. $\gg-$; les temps de travail, soit le nombre total d'heures ou la flexibilité horaire - « J'ai priorisé les heures de travai $1^{14}$. $\gg-$; et les relations dans le travail - « Je pense que l'ambiance est le plus important, le groupe de gens surtout ${ }^{15}$. »

À partir de ces critères, pour accepter un emploi, y rester ou le quitter, les jeunes construisent leur propre grille de définition et de sélection des emplois. Par ailleurs, les critères du rapport à l'emploi ont une valeur relative : un meilleur salaire a la priorité si les horaires conviennent, la sécurité de l'emploi est souhaitée si cela n'implique pas une forte diminution du salaire...

Cependant, sur des marchés du travail - tels que l'Argentine - où l'emploi informel constitue pour les jeunes la principale voie d'accès à l'emploi, l'intégration ne peut pas être pensée seulement rattachée au modèle salarial typique. Serge Paugam (2000) propose d'établir une distinction entre rapport au travail et rapport à l'emploi en vue de l'analyse de l'intégration professionnelle. La première notion implique les dimensions de satisfaction et d'insatisfaction des salariés dans l'exercice de leur activité alors que la deuxième indique le degré de stabilité de leur situation professionnelle et l'engagement collectif des salariés. Le rapport au travail rendrait compte des formes d'aliénation dépassant l'organisation sociale du travail, alors que le rapport à l'emploi renverrait aux formes d'exploitation (Paugam, 2000) propres à un système donné.

\footnotetext{
9. Luciano, formation professionnelle, vague 2.

10. Sandra, polimodal privé, vague 1.

11. Darío, polimodal public, vague 1 .

12. Gustavo, technique privé, vague 1.

13. Raúl, polimodal public, vague 1.

14. María, polimodal privé, vague 2 .

15. Javier, polimodal privé, vague 2 .
} 
Dossier

Rapports des jeunes au travail, pratiques d'emploi et diplômes

\section{Le rapport au travail : finalités mobilisées pour travailler}

La notion de rapport au travail se rapporte à ce qui lie les sujets au travail au-delà de son système d'organisation sociale et historique dans un contexte de changements profonds du monde du travail. En effet, les crises contemporaines du travail sont d'abord des crises du travail salarié (Bouffartigue, Eckert, 1997). Christian Baudelot et Michel Gollac (2003) montrent qu'il existe une pluralité de sources de bonheur et de malheur dans les emplois. Le vécu et la perception du travail sont plus étendus que l'activité prescrite. Les auteurs distinguent ainsi différents registres du plaisir (les contacts humains, les occupations, la dimension créatrice du travail, la découverte).

Le caractère positif ou négatif des registres incitant les jeunes à travailler varie en fonction du moment de leurs parcours, ce qui nous amène à abandonner ici les termes «bonheur » et « malheur » utilisés par ces auteurs pour étudier le rapport au travail. De plus, s'agissant de jeunes, l'accent a été mis notamment sur les raisons qui motivent leur première insertion. Ces derniers en énoncent toute une série : la rétribution ou l'obtention de revenus - « Je travaille parce que j'aime l'argent ${ }^{16}$. » - ; l'autonomie vis-àvis des adultes, la famille d'origine ou les adultes en général - «Travailler implique que je ne dépends que de moi-même ${ }^{17}$. " - ; l'épanouissement ou le développement des compétences et des intérêts constituant l'identité propre et l'estime de soi - «Travailler est une manière d'accorder une dignité à la personne ${ }^{18}$. »- ; l'expérience professionnelle et l'expérience de vie en général - « Je travaille surtout pour commencer à gagner en expérience $^{19}$. $\gg-;$; la recherche de liens entre formation et travail et la mise en œuvre des compétences et des qualifications acquises lors d'une formation - « Je n'ai rien vu de tout ça à l'école et il y avait cette option [travailler] pour me l'apprendre ${ }^{20}$. »Ces finalités sont multiples et variées : il est rare qu'un jeune affirme travailler pour une seule et unique raison, même si les motifs varient d'un individu à l'autre et quelquefois, chez un même jeune, tout au long du parcours.

Cette liste de raisons pour intégrer le marché du travail trouve son intérêt dans le fait qu'en partant des énoncés individuels, il est possible de reconstruire des finalités historiquement attribuées au travail : instrumentales, expressives, formatives, sociales, etc. Ces finalités coexistent empiriquement, dans une plus ou moins large mesure, témoignant de la complexité du travail.

\footnotetext{
16. Javier, polimodal privé, vague 2 .

17. Celeste, formation professionnelle, vague 2.

18. María, polimodal privé, vague 2 .

19. Guillermina, polimodal public, vague 1 .

20. Rocío, technique public, vague 2 .
} 


\section{Le rapport au monde professionnel : tamis des opportunités}

Une dernière dimension avec des antécédents moindres ${ }^{21}$ est le rapport au monde professionnel. Celui-ci est associé au marché du travail et à la façon dont les jeunes le perçoivent. «Concernant les déterminants de l'insertion, les "causes" n'ont pas d'"effets" en soi tant les "logiques de construction" médiatisent l'impact des facteurs et en modulent le sens » (Nicole-Drancourt, 1994, p. 64). Le regard que les jeunes portent sur le marché du travail configure la conscience des opportunités de travail, qui vont structurer, stimuler ou limiter leur recherche d'emploi. En effet, au moment d'effectuer des choix, les individus tendent à négliger certaines données en fonction de l'intérêt (Savard et al., 2007) et des informations qu'ils en ont (Gallant et al., 2016).

Au moment où les jeunes du panel acceptaient de participer à l'étude, en 2006, le contexte économique argentin était marqué par le début d'un intense remaniement. Malgré ce contexte similaire pour tous, deux positionnements extrêmes ont été identifiés : des jeunes qui perçoivent un monde d'opportunités et d'autres qui considèrent que le contexte n'en offre pas. Des déclinaisons à l'intérieur de ces pôles sont possibles, emphatisant certains aspects du marché du travail : des opportunités individuelles qui dépendent de l'effort personnel - « Je pense qu'il y a des opportunités, il importe à chacun de les chercher ${ }^{22}$. »-; des opportunités sociales ou généralisées ouvrant des portes ${ }^{23}$ - «Les techniciens sont très sollicités, car il est en train de se mettre en place beaucoup de technologies ${ }^{24}$. $\gg-$; le caractère exigeant en termes de diplôme, d'expérience, d'âge et de conditions de travail - «C'est dur de trouver un emploi, ils sont exigeants, ils te demandent l'école primaire, secondaire ; avant c'était différent ${ }^{25}$. »- ; le caractère précaire ou un marché du travail qui est vu comme un espace d'aliénation, créant de mauvaises conditions pour travailler - «C'est assez nébuleux, car la majorité des emplois sont non déclarés, temporaires. En même temps, les emplois déclarés, si tu regardes, tu vas travailler dans une usine et, du jour au lendemain, ils te disent qu'ils doivent licencier des gens et ils te licencient ${ }^{26}$. »

Les jeunes perçoivent donc de façon distincte le monde du travail qui les entoure et les opportunités que ce dernier leur offre. Le fait de retrouver des jeunes issus de divers types de formation en regard des différents points de vue montre que le rapport au monde professionnel n'est pas uniquement tributaire de la condition sociale, mais que la médiation symbolique y joue également un rôle important.

21. Si ce n'est pas à travers l'avis des travailleurs sur les modes de gestion (Mercure, Vultur, 2010).

22. Julia, polimodal public, vague 1.

23. À partir de 2006, l'amélioration des conditions de production a augmenté la demande d'emploi et le besoin de techniciens dans certains secteurs.

24. Miguel, technique public, vague 1.

25. Gimena, formation professionnelle, vague 1.

26. Carla, formation professionnelle, vague 2 . 
Dossier

Rapports des jeunes au travail, pratiques d'emploi et diplômes

En somme, la quête d'association d'éléments symboliques et de pratiques liés au travail, le besoin de préserver et en même temps d'unifier la diversité des dimensions de la vie professionnelle, la nécessité de montrer la multiplicité des positionnements des individus socialement situés, l'exigence de contextualiser l'insertion tant pour le groupe spécifique des jeunes que pour un cadre sociétal particulier - comme le contexte argentin - ont conduit à élaborer la notion de rapports à la vie professionnelle.

Les rapports à la vie professionnelle étudiés chez les jeunes de l'enquête synthétisent ceux à l'activité, au travail, à l'emploi et au monde professionnel, définissant la force et la forme de l'engagement avec lesquelles l'individu s'assume en tant que travailleur. Tous ces aspects liés à la vie professionnelle font donc partie d'un même objet, mais leur poids varie selon les autres caractéristiques du parcours et les différents moments.

\section{LA DYNAMIQUE DES RAPPORTS À LA VIE PROFESSIONNELLE}

La construction des rapports à la vie professionnelle commence pendant l'enfance, constituant les ressources héritées et les rapports familiaux (Nicole-Drancourt, 1994). Mais, a priori, ces constructions sont toujours en cours d'élaboration au moment de l'insertion professionnelle. Elles ne sont pas non plus définitivement achevées au moment du premier emploi ; elles se façonnent tout au long du processus d'insertion (Bidart, Longo, 2007). Elles s'élaborent dans des mondes professionnels de référence (Demazière, Dubar, 1997) ou dans des secteurs ayant leurs logiques propres (Loriol, 2017).

Les rapports à la vie professionnelle sont donc susceptibles de se modifier avec le temps. Ils s'édifient dans le passé, et se projettent dans l'avenir. Leur compréhension dynamique devient cruciale dans le contexte actuel de réversibilité des parcours (Hamel, 2003 ; Côté, 2013), et encore davantage dans un contexte social instable caractérisé en Argentine par l'alternance de phases courtes de croissance, de récessions et de crises aiguës.

Grâce aux données longitudinales de l'enquête argentine, il est possible d'observer la récurrence de certaines des évolutions de ces rapports : une plus grande importance accordée au travail d'une vague à l'autre ${ }^{27}$; une variation importante des raisons instrumentales de travailler vers des raisons expressives et identitaires ${ }^{28}$; la persistance de la sécurité, de la tâche, du salaire et du temps de travail comme des critères clés; et la réduction progressive d'opportunités, remarquée par les jeunes à la suite d'une plus grande fréquentation du marché du travail.

27. En vague 3, la centralité de l'activité caractérise la moitié des rapports (24/46).

28. En vague 3, 22/46 rapports sont dominés par l'épanouissement et 19/46 par la rétribution. 


\section{L'INTERACTION DES RAPPORTS, DES PRATIQUES ET DES DIPLÔMES}

La grande majorité des travaux portant sur les rapports au travail ne rendent pas compte des interactions entre représentations symboliques et actions effectives, et encore moins entre leurs évolutions respectives. L'analyse des rapports à la vie professionnelle est pourtant féconde dans la mesure où elle permet d'améliorer la compréhension des parcours concrets des jeunes, tout en permettant de déceler le rôle de l'origine sociale ou du diplôme.

En observant simplement les trajectoires objectives d'emploi des jeunes du panel, nous pouvons les classer selon quatre types : « stabilisation immédiate »; « stabilisation différée »; « instabilité professionnelle »; « inactivité continue ». Au premier abord, ces trajectoires ne sont pourtant pas exclusives d'une seule catégorie éducative ou sociale. La même trajectoire se retrouve chez des jeunes ayant des origines sociales et scolaires distinctes. Cependant, ce dernier constat mettant en exergue la différenciation des parcours peut être nuancé à l'aide des rapports à la vie professionnelle. En effet, à travers ces derniers, il est possible de mieux comprendre la manière dont le niveau ou le type de diplôme de même que l'origine sociale s'associent non seulement aux pratiques des parcours, mais aussi à la subjectivité engagée, créant différentes possibilités de poursuite du parcours. Les résultats qui suivent illustrent ces associations au sein des parcours d'emploi des jeunes.

Tableau 1. Trajectoires objectives des jeunes du panel argentin (vagues 1 et 2)

\begin{tabular}{|c|c|c|c|c|c|c|}
\hline 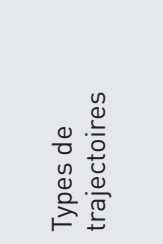 & 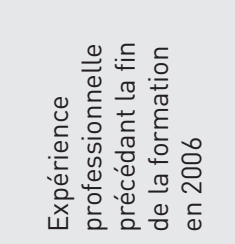 & 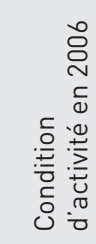 & 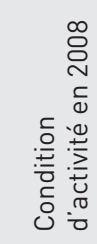 & 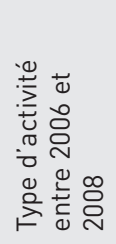 & 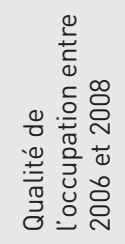 & 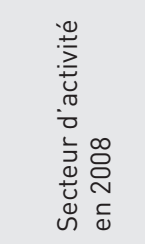 \\
\hline $\begin{array}{l}\text { Stabilisation } \\
\text { immédiate }\end{array}$ & $\begin{array}{l}\text { Sans expérience } \\
\text { ou expérience } \\
\text { des petits boulots }\end{array}$ & Inactif & Actif & $\begin{array}{l}\text { Stabili- } \\
\text { sation }\end{array}$ & $\begin{array}{l}\text { Emploi } \\
\text { formel }\end{array}$ & $\begin{array}{l}\text { Commerce } \\
\text { Industrie } \\
\text { Services }\end{array}$ \\
\hline $\begin{array}{l}\text { Inactivité } \\
\text { continue }\end{array}$ & $\begin{array}{l}\text { Sans expérience } \\
\text { ou expérience } \\
\text { des petits boulots }\end{array}$ & Inactif & Inactif & $\begin{array}{l}\text { Inacti- } \\
\text { vité }\end{array}$ & $\begin{array}{l}\text { Emploi } \\
\text { informel }\end{array}$ & Variés \\
\hline $\begin{array}{l}\text { Stabilisation } \\
\text { différée }\end{array}$ & $\begin{array}{l}\text { Expérience dans } \\
\text { des emplois } \\
\text { permanents, } \\
\text { temporaires et } \\
\text { des petits boulots }\end{array}$ & Actif & Actif & $\begin{array}{l}\text { Stabili- } \\
\text { sation }\end{array}$ & $\begin{array}{l}\text { Emploi } \\
\text { formel/ } \\
\text { informel }\end{array}$ & $\begin{array}{l}\text { Industrie } \\
\text { Bâtiment } \\
\text { Commerce } \\
\text { Services }\end{array}$ \\
\hline $\begin{array}{l}\text { Instabilité } \\
\text { profession- } \\
\text { nelle }\end{array}$ & $\begin{array}{l}\text { Expérience dans } \\
\text { des emplois } \\
\text { temporaires et } \\
\text { des petits boulots }\end{array}$ & Actif & Actif & $\begin{array}{l}\text { Forte } \\
\text { rotation } \\
\text { et } \\
\text { mobilité }\end{array}$ & $\begin{array}{l}\text { Emploi } \\
\text { informel }\end{array}$ & $\begin{array}{l}\text { Services } \\
\text { Industrie }\end{array}$ \\
\hline
\end{tabular}


Dossier

Rapports des jeunes au travail, pratiques d'emploi et diplômes

Tableau 2. Distribution des trajectoires objectives des jeunes du panel selon le diplôme et le sexe (vagues 1 et 2)

\begin{tabular}{|c|c|c|c|c|c|}
\hline $\begin{array}{l}\text { Trajectoires } \\
\text { objectives }\end{array}$ & $\begin{array}{l}\text { Polimodal } \\
\text { privé }\end{array}$ & $\begin{array}{l}\text { Polimodal } \\
\text { public }\end{array}$ & $\begin{array}{l}\text { Technique } \\
\text { privé }\end{array}$ & $\begin{array}{l}\text { Technique } \\
\text { public }\end{array}$ & $\begin{array}{l}\text { Formation } \\
\text { profes- } \\
\text { sionnelle }\end{array}$ \\
\hline $\begin{array}{l}\text { Stabilisation } \\
\text { immédiate }\end{array}$ & $\begin{array}{l}0^{\pi} 0^{\pi} 0^{\pi} \\
\text { 우우우우우 }\end{array}$ & $\begin{array}{l}\hat{0} 0 \hat{0} \\
\text { 우웅 }\end{array}$ & के & $\begin{array}{l}30 \\
90 \\
9+9\end{array}$ & 우우 \\
\hline Inactivité continue & $\begin{array}{l}3 \pi \\
\text { 90 } \\
+9\end{array}$ & $\begin{array}{l}1 \pi \\
01 \\
\text { 99+9 }\end{array}$ & 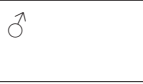 & $\begin{array}{l}1 \lambda \pi \\
019 \\
\text { 우 }\end{array}$ & 우우 \\
\hline $\begin{array}{l}\text { Stabilisation } \\
\text { différée }\end{array}$ & $\begin{array}{l}0 \\
0 \\
0 \\
\end{array}$ & $\begin{array}{l}\hat{0} \\
\text { o } \\
\end{array}$ & के के & 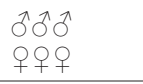 & $\begin{array}{l}30 \pi \\
0 \\
0\end{array}$ \\
\hline $\begin{array}{l}\text { Instabilité } \\
\text { professionnelle }\end{array}$ & $\begin{array}{l}\text { 허 } \\
\text { 우우오 }\end{array}$ & 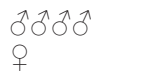 & $\sigma^{\lambda}$ & $\hat{\partial} \widehat{O}$ & 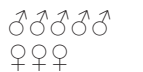 \\
\hline
\end{tabular}

- La trajectoire de « stabilisation immédiate » est définie par l'absence d'expérience professionnelle et par l'inactivité à la sortie de la formation, suivies d'une insertion directe et stable. Elle caractérise particulièrement les parcours des jeunes tant de la filière polimodal que technique, privée et publique. Toutefois, si ces différents types de formation partagent la même trajectoire, elle revêt des sens différents pour les uns et pour les autres. D'une part, les jeunes issus des écoles privées (soit polimodal ou technique) ou provenant d'un milieu aisé et intermédiaire connaissent une évolution dans leurs rapports à l'activité : ils passent de la non-centralité à la propension à l'activité ou à sa centralité - si on reprend les modalités développées plus haut - ; ils travaillent pour des raisons instrumentales et ils s'attachent à des critères d'emploi fortement liés au modèle salarial ainsi qu'à la vision d'un monde professionnel précaire et exigeant. D'autre part, les jeunes issus des écoles techniques et d'origines intermédiaires et populaires ne donnent pas la priorité à l'activité professionnelle, mais plutôt à l'association formation-emploi. Ils s'attachent aux critères de la tâche pour choisir leurs emplois et considèrent le monde professionnel comme un monde offrant des opportunités sociales et individuelles.

- La trajectoire de «stabilisation différée » est définie par une importante expérience professionnelle sous forme d'emplois permanents, temporaires et de petits boulots, commencée avant même la fin de la formation, poursuivie ensuite par une stabilisation ultérieure dans des emplois déclarés ou non. Elle caractérise les parcours des jeunes des écoles techniques et de la formation professionnelle. Pourtant, ces deux groupes se différencient, notamment en fonction de leurs rapports à la vie professionnelle. D'un côté, les jeunes du premier groupe, principalement issus des écoles privées, donnent la priorité à l'activité professionnelle dans leur vie ; l'épanouissement est pour eux la principale raison de travailler. Ils n'ont pas d'exigences 
en matière de critères d'emploi et appréhendent le monde professionnel comme un monde d'opportunités soit individuelles soit sociales. Le deuxième groupe a un rapport à l'activité caractérisé par la naturalisation, voire l'absence de remise en question du travail dans leur vie. La principale raison qui les motive à travailler est la rétribution et ils s'attachent aux critères de l'emploi salarié dans un monde professionnel qui comporte à la fois des opportunités et des exigences.

- La trajectoire d'« instabilité professionnelle » est définie par une grande expérience professionnelle d'emplois temporaires et de petits boulots, acquise avant même la fin de la formation, suivie d'une insertion marquée par l'entrée et la sortie du marché du travail et par une succession d'emplois non déclarés et instables. Elle caractérise les parcours des jeunes, notamment issus de la formation professionnelle et du secondaire polimodal, public ou privé. Ces mondes sociaux s'écartent l'un de l'autre pourtant lorsque l'on observe les rapports à la vie professionnelle. D'une part, se trouvent les jeunes, principalement de classe moyenne, issus de la filière polimodal, publique ou privée, qui, ayant une propension à travailler, voient leur motivation évoluer : ils passent d'une vision instrumentale à une vision expressive du rapport au travail, tout en considérant le monde professionnel comme précaire. D'autre part, les jeunes d'origine populaire, issus de la formation professionnelle ou du secondaire technique public, ont des rapports à l'activité, au travail, à l'emploi et au monde professionnel qui évoluent face à la précarité et aux difficultés d'insertion et de stabilisation. Ils passent ainsi de la centralité de l'activité professionnelle à son aversion, du travail pour l'autonomie au travail pour la rétribution, de l'intérêt porté au critère de la tâche à un intérêt centré sur la stabilité et la sécurité de l'emploi, d'une vision du monde professionnel offrant des opportunités à celle plutôt caractérisée par la précarité.

- La trajectoire d'« inactivité continue » est définie par l'absence d'expérience professionnelle et l'inactivité dominante tout au long de la période. Elle caractérise les parcours des jeunes des écoles publiques, principalement de la filière polimodal de même que ceux des jeunes de la formation professionnelle. Cependant, cette situation objective se précise si nous considérons les rapports à la vie professionnelle. Nous observons, d'un côté, des jeunes qui retardent leur insertion dans le monde du travail en raison d'une formation ou de la maternité, car ils ne donnent pas la priorité à l'activité professionnelle. La rétribution constitue la principale raison de travailler à ce moment précis de leur parcours de vie et ils considèrent le monde professionnel comme exigeant et précaire. Ce positionnement est partagé tant 
Dossier

Rapports des jeunes au travail, pratiques d'emploi et diplômes

Tableau 3. Trajectoires objectives des jeunes du panel selon les évolutions des rapports à la vie professionnelle (vagues 1 et 2)

\begin{tabular}{|c|c|c|c|c|c|c|c|c|}
\hline 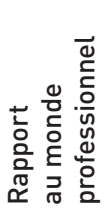 & 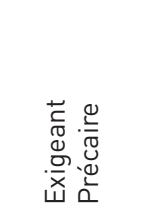 & 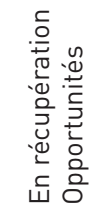 & 离 & 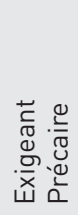 & 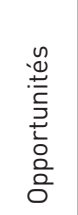 & 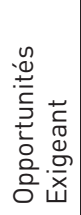 & 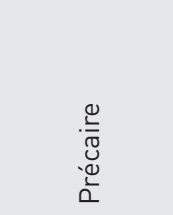 & 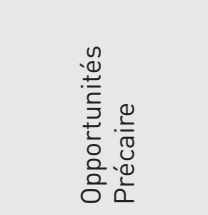 \\
\hline 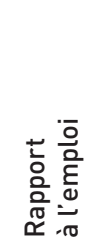 & 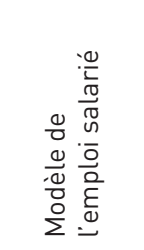 & 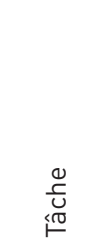 & 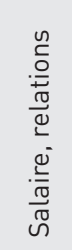 & 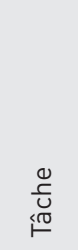 & $\begin{array}{l}\frac{01}{\frac{1}{L}} \\
\stackrel{5}{>}\end{array}$ & 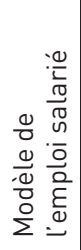 & 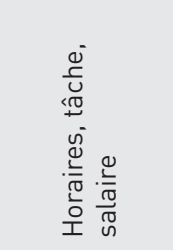 & 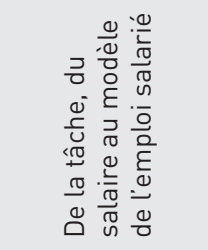 \\
\hline 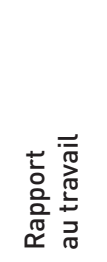 & 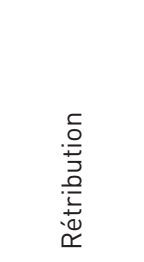 & 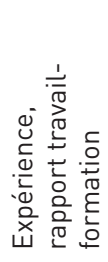 & 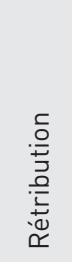 & 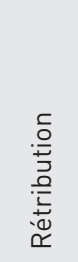 & 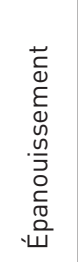 & 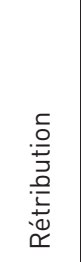 & 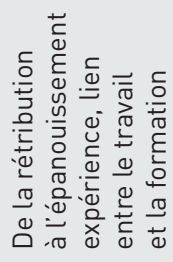 & 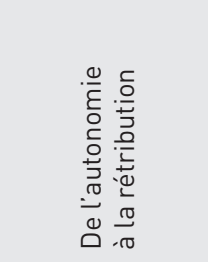 \\
\hline 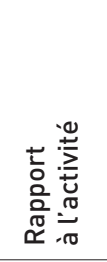 & 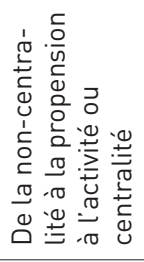 & 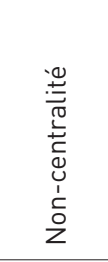 & $\begin{array}{l}\frac{c}{0} \\
\frac{0}{0} \\
\frac{0}{4}\end{array}$ & 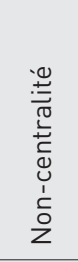 & 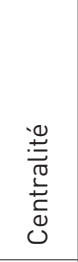 & 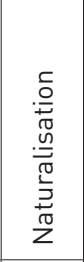 & 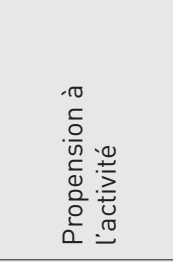 & 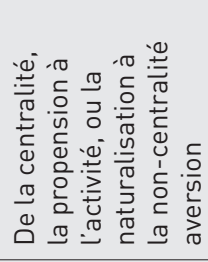 \\
\hline 竞 & 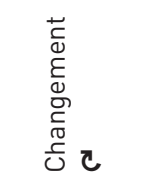 & 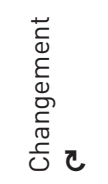 & 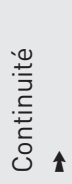 & 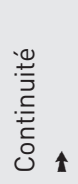 & 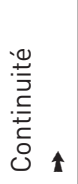 & 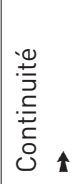 & 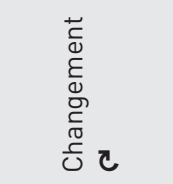 & 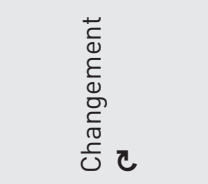 \\
\hline & 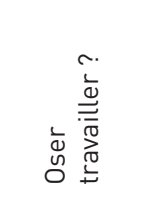 & 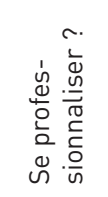 & 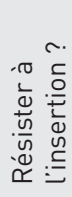 & 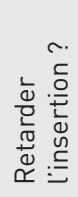 & 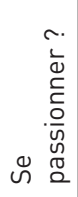 & 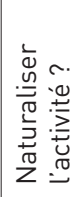 & 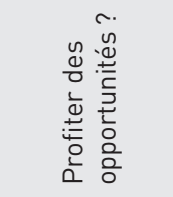 & 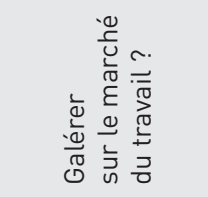 \\
\hline 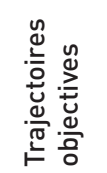 & \multicolumn{2}{|c|}{ 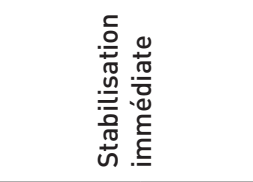 } & \multicolumn{2}{|c|}{ 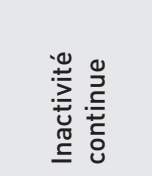 } & \multicolumn{2}{|c|}{ 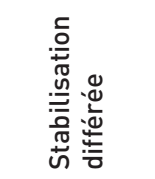 } & \multicolumn{2}{|c|}{ 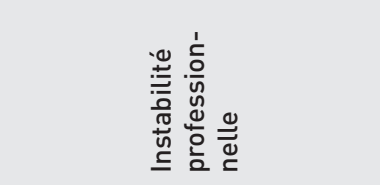 } \\
\hline
\end{tabular}


par les jeunes de la filière polimodal publique ou privée que par les jeunes de milieu populaire appartenant à la filière technique du secteur public ou à la formation professionnelle. Par ailleurs, cette situation objective s'explique par une «aversion » explicite de l'activité professionnelle qui va de pair avec un choix pour les études longues. Il s'agit ici de jeunes issus de milieux aisés et intermédiaires, de même que de jeunes du secondaire polimodal privé.

Tableau 4. Distribution des parcours des jeunes du panel selon le diplôme et le sexe (vagues 1 et 2)

\begin{tabular}{|c|c|c|c|c|c|}
\hline $\begin{array}{l}\text { Parcours des jeunes } \\
\text { (trajectoires objectives } \\
\text { + rapports à la vie } \\
\text { professionnelle } \\
\text { + évolutions) }\end{array}$ & $\begin{array}{l}\text { Polimodal } \\
\text { privé }\end{array}$ & $\begin{array}{l}\text { Technique } \\
\text { privé }\end{array}$ & $\begin{array}{l}\text { Polimodal } \\
\text { public }\end{array}$ & $\begin{array}{l}\text { Technique } \\
\text { public }\end{array}$ & FP \\
\hline Oser travailler? & 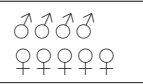 & 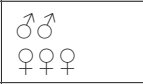 & $\hat{\partial} \hat{O}$ & 우 & 우우 \\
\hline Se professionnaliser? & & & $\hat{\partial O}$ & $\begin{array}{l}\hat{o}^{\pi} 0^{2} \\
+\end{array}$ & \\
\hline Résister à l'insertion? & 우우 & & & $\pi$ & \\
\hline Retarder l'insertion? & $\hat{O} \hat{O}^{-1}$ & $\begin{array}{l}0 \pi \\
00 \\
+9+9 \\
\end{array}$ & o & $\begin{array}{l}\hat{0} \\
\text { 우우 }\end{array}$ & qq+ \\
\hline Se passionner? & $\begin{array}{l}0 \\
0 \\
+\end{array}$ & q & $\hat{\partial}{ }^{\lambda}$ & $\pi$ & \\
\hline Naturaliser l'activité ? & & $0^{\lambda}$ & $0^{\pi}$ & $\begin{array}{l}\hat{0} 0 \hat{0} \\
\text { 우우 }\end{array}$ & 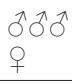 \\
\hline $\begin{array}{l}\text { Profiter des } \\
\text { opportunités? }\end{array}$ & $\begin{array}{l}0 \hat{0} \\
\text { 웅 } \\
\end{array}$ & $\widehat{\partial} \delta^{\lambda}$ & $0^{\pi}$ & $\hat{0}$ & $\begin{array}{l}0 \hat{0} \hat{0}^{-1} \\
\text { +9 }\end{array}$ \\
\hline $\begin{array}{l}\text { Galérer sur le marché } \\
\text { du travail ? }\end{array}$ & & $\begin{array}{l}0 \\
0 \\
+\end{array}$ & & $\pi$ & $\begin{array}{l}\hat{0} 0^{\pi} \\
\text { 우 }\end{array}$ \\
\hline
\end{tabular}

Au regard de ce qui précède, la subjectivité au travail est amalgamée avec d'autres facteurs nous permettant d'y retrouver l'origine sociale ou le diplôme qui, conjugués aux pratiques effectives, permettent de comprendre l'orientation des parcours, malgré leur apparente similarité ou diversité. Il reste toutefois à approfondir le mode, le comment, de ces interactions. Par ailleurs, cet amalgame doit être analysé dans une perspective dynamique : ce sont parfois les évolutions et pas seulement le positionnement des rapports à la vie professionnelle qui montrent la raison pour laquelle un jeune emprunte une voie plutôt qu'une autre. Il en est de même pour le diplôme par exemple, dont la valeur peut évoluer en fonction des difficultés d'insertion, devenant symboliquement « inefficace » pour certains jeunes. Enfin, si tout n'est pas fixé d'avance par les variables d'origine des parcours, il n'en 
Dossier

Rapports des jeunes au travail, pratiques d'emploi et diplômes

reste pas moins que les marges de manœuvre des jeunes se construisent suivant une distribution sociale et personnelle des sens. Les parcours se différencient donc non seulement en fonction de l'hétérogénéité des conditions d'emplois ou de la structure sociale, mais également en raison de la pluralité de significations que les jeunes donnent à la vie professionnelle.

Ces significations suggèrent également quelques pistes pour les interventions auprès des jeunes. Dans un contexte de perte de droits et de protections sociales, le rapport à l'emploi montre que le modèle d'emploi salarial typique (symbole de sécurité, de stabilité et de carrière) ne constitue pas toujours un critère central chez les jeunes, du moins, pas à n'importe quel prix. Cette vision ne va toutefois pas à l'encontre de l'effort politique d'améliorer les conditions de travail des jeunes, mais vise aussi à adapter les dispositifs à leurs critères. Pour ce qui est du rapport à l'activité, celui-ci incite à porter un regard holistique sur les parcours et à imaginer des dispositifs de conciliation entre le travail, les études et la famille, en même temps qu'il remet en question les théories sur la perte de la centralité du travail dans la vie des jeunes. Quant au rapport au travail, il contredit l'idée que dans un contexte de précarisation et de dégradation des conditions de travail, celui-ci semblerait se départir de ses fonctions expressives et identitaires. En outre, le rapport au monde professionnel montre la nécessite de prévenir une vision erronée du marché du travail, en améliorant l'information et son accès pour les différentes catégories de jeunes.

\section{BIBLIOGRAPHIE}

Antunes R., 2005, Los sentidos del trabajo. Ensayo sobre la afirmación y la negación del trabajo, Ediciones Herramienta, Taller de Estudios Laborales, Buenos Aires (Argentine).

Baudelot C., Gollac M., 2003, Travailler pour être heureux ? Le bonheur et le travail en France, Paris, Fayard.

Bernoux P., 2016, « Un changement de paradigme, le travail comme activité », Sociologie et sociétés, n 1, vol. 48, p. 15-34.

BidART C., Longo M. E., 2007, «Bifurcations biographiques et évolutions des rapports au travail », in Giret J.-F., GRelet Y., LAvialle C., Timoteo J., WeRQUIN P. (dir.), Rupture et irréversibilités dans les trajectoires, CEREQ/ Relief, $n^{\circ} 22$, Marseille. 
Bouffartigue P., Eckert H. (dir.), 1997, Le travail à l'épreuve du salariat. À propos de la fin du travail, Paris, L'Harmattan.

Côté N., 2013, «Pour une compréhension dynamique du rapport au travail : la valeur heuristique de la perspective des parcours de vie », Sociologie et sociétés, n 1, vol. 45, printemps 2013, p. 179-201.

DaUne-RICHARD A.-M., 1992, « Trajectoires de socialisation et rapport à l'emploi », Revue française des affaires sociales, $n^{\circ} 2$, avril-juin, p. 85-95.

Delay B., 2008, Les jeunes : un rapport au travail singulier? Une tentative pour déconstruire le mythe de l'opposition entre les âges, Document du CEE, n 104, septembre.

Demazière D., Dubar C., 1997, Analyser les entretiens biographiques. L'exemple des récits d'insertion, Nathan, Paris.

Dubar C., 1994, "L'insertion comme articulation temporelle du biographique et du structurel », Revue française de sociologie, n 35, p. 283-291.

Dubet F., 2000, Les inégalités multipliées, Éditions de l'Aube.

Dubet F., Duru-Bellat M., Vérétout A., 2010, Les sociétés et leur école. Emprise du diplôme et cohésion sociale, Le Seuil, Paris.

Eckert H., Vultur M., 2016, «Présentation. Activité et circonstances de l'activité », Sociologie et sociétés, nº 1, vol. 48, p. 5-12.

EGRIS (European Group for InTEgrated Social Research), 2000, « Trayectorias encauzadas o no encauzadas? », Propuesta educativa, año $10, n^{\circ} 23$, décembre.

Filmus D., Kaplan C., Miranda A., Moragues M., 2001, Cada vez más necesaria, cada vez más insuficiente : escuela media y mercado de trabajo en épocas de globalización, Santillana, Buenos Aires (Argentine).

Gallant N., Supeno E., Atkin S., Labrecque K., Cardona J., Longo M. E., 2016, Pratiques informationnelles en matière d'insertion en emploi. Le cas des jeunes et des immigrants récents au Québec, Rapport remis au ministère du travail, de l'emploi et de la solidarité sociale, décembre.

GAUTIÉ J., 2003, «Transition et trajectoires sur le marché du travail », Quatre pages, Centre d'études et de l'emploi (CEE), n59, septembre.

Hamel J., 2003, «Pour une vue longitudinale sur les jeunes et le travail », Cahiers internationaux de sociologie, $\mathrm{n}^{\circ} 115$, vol. 2, p. 255-268.

Jacinto C., Chitarroni H., 2010, « Precariedades, rotación y movilidades en las trayectorias laborales juveniles », Estudios del trabajo, n 39-40, p. 5-36.

LAHIRE B., 1998, L'homme pluriel. Les ressorts de l'action, Armand Colin, Paris. Lima L., 2016, Pauvres jeunes. Enquête au cœur de la politique sociale de jeunesse, Champ social, Paris. 
Rapports des jeunes au travail, pratiques d'emploi et diplômes

LoNGo M. E., 2014, « Co-construire l'insertion professionnelle des jeunes », in Les dispositifs publics en action. Vers une sécurisation des parcours professionnels et personnels ? Éditions Liaisons, Rueil-Malmaison, p. 81-99.

Longo M. E., 2016a, «Réguler aussi avec l'informel. L'informalisation de l'État au cœur de l'emploi des jeunes », Lien social et politiques, $n^{\circ} 76$, p. 178-205.

LoNGo M. E., 2016b, « Les parcours de vie des jeunes comme des processus », Les cahiers dynamiques, $n^{\circ} 67$, «Parcours de jeunes et institutions », p. 48-57.

Longo M. E., 2016c, « L'âge éphémère. Les définitions de la jeunesse à la lumière du temps », Revue jeunes et société, n 1, vol. 1, p. 5-24.

Longo M. E., Bourdon S., 2016, « La configuration en "deux temps" des rapports à la vie professionnelle. Une particularité des jeunes à l'aube des études postsecondaires au Québec », SociologieS [en ligne], «Théorie et recherche », 19 octobre 2016.

LORIOL M., 2017, Le(s) rapport(s) des jeunes au travail. Revue de littérature (2006-2016), rapport d'étude, INJEP.

MÉdA D., 2010, « Comment mesurer la valeur accordée au travail ? », Sociologie [en ligne], $\mathrm{n}^{\circ} 1$, vol. 1.

Méda D., Vendramin P., 2013, Réinventer le travail, Paris, Presses universitaires de France.

Mercure D., Vultur M., 2010, La signification du travail. Nouveau modèle productif et ethos du travail au Québec, Presses de l'Université Laval, Sainte-Foy (Canada).

MTEyss (Ministerio de Trabajo, Empleo y Seguridad Social de Argentina), 2005, Trabajo, ocupación y empleo. Trayectorias, negociación colectiva e ingresos, agosto, Subsecretaría de Programación Técnica y Estudios Laborales.

Miranda A., 2006, Desigualdad educativa e inserción laboral segmentada de los jóvenes en la Argentina contemporánea. Thèse de doctorat, FLACSO-Facultad Latinoamaricana de Ciencias Sociales, Buenos Aires (Argentine).

Nicole-Drancourt C., 1992, « Mode de socialisation et rapport à l'activité », Revue française des affaires sociales, $n^{\circ} 2$, avril-juin, p. 71-83.

Nicole-Drancourt C., 1994, «Mesurer l'insertion professionnelle 》, Revue française de sociologie, $\mathrm{n}^{\circ}$ 35, p. 37-68.

Nicole-Drancourt C., Roulleau-Berger L., 2001, Les jeunes et le travail. 1950-2000, Presses universitaires de France, Paris.

Passeron J.-C., 1990, « Biographies, flux, itinéraires, trajectoires », Revue française de sociologie, $n^{\circ} 1$, vol. 31, p. 3-22. 
Paugam S., 2000, Le salarié de la précarité, Presses universitaires de France, Paris.

Pérez P., 2008, La inserción ocupacional de los jóvenes en un contexto de desempleo masivo. El caso argentino entre 1995 y 2003, Miño y Davila Editores/Ceil-Piette, Buenos Aires (Argentine).

Pérez P., Deleo C., Fernández Massi M., 2016, « Une insertion professionnelle inégale : l'importance de l'origine sociales dans les transitions professionnelle des jeunes en Argentine », Revue jeunes et société, $n^{\circ} 2$, vol. 1, p. 29-56.

Roberts S., Clark S. C., Wallace C., 1994, « Flexibility and individualisation : a comparison of transitions into employment in England and Germany », Sociology, n 1, vol. 28, p. 31-54.

Salvia A., 2013, Juventudes, problemas de empleo y riesgos de exclusión social. El actual escenario de crisis mundial en la Argentina, Friedrich Ebert Stiftung, Berlin (Allemagne).

Salvia A., TuÑon I., 2003, Los jóvenes trabajadores frente a la educación, el desempleo y el deterioro social en la Argentina, Fundación Hebert, Buenos Aires (Argentine).

Savard R., Michaud G., Bilodeau C., Arseneau S., 2007, « L'effet de l'information sur le marché du travail dans le processus décisionnel relatif au choix de carrière », Canadian Journal of Counselling and Psychoterapy, no 3, vol. 41, p. 158-172.

SPReW, 2008, Generational Approach to the Social Patterns of Relation to Work, Rapport de synthèse final.

\section{L'AUTEURE}

\section{María Eugenia Longo}

Maria-Eugenia.Longoducs.inrs.ca

Professeure à l'Institut national de la recherche scientifique (INRS), Urbanisation Culture Société (UCS), Québec (Canada), directrice de l'Observatoire jeunes et société. Thèmes de recherche : travail ; parcours ; jeunes ; temporalités ; politiques.

\section{A notamment publié}

Longo M. E., 2016, « Réguler aussi avec l'informel. L'informalisation de l'État au cœur de l'emploi des jeunes », Lien social et politiques, $n^{\circ}$ 76, p. 178-205.

Longo M. E., 2016, « L'âge éphémère. La définition de la jeunesse à la lumière du temps », Revue jeunes et société, no 1, vol. 1, p. 5-24.

LoNGo M. E., 2015, "Youth temporalities and uncertainty : understanding variation in young Argentinians' professional careers », Time and Society (doi:10.1177/0961463X15609828). 